\title{
Alterations of the gastric stump and not resected stomach mucosa after truncal vagotomy in rats ${ }^{1}$
}

\author{
Paulo Antônio Rodrigues ${ }^{I}$, Luiz Eduardo Naresse ${ }^{\mathrm{II}}$, José Guilherme Minossi ${ }^{\mathrm{III}}$, Claudia Nishida Hasimoto ${ }^{\mathrm{IV}}$
}

${ }^{I} \mathrm{PhD}$, Assistant Professor, Departament of Surgery \& Orthopedics, Faculty of Medicine, Sao Paulo State University (UNESP), Botucatu-SP, Brazil. Conception, design, intellectual and scientific content of the study; acquisition and interpretation of data; technical procedures; manuscript writing. IIPhD, Associate Professor, Departament of Surgery \& Orthopedics, Faculty of Medicine, UNESP, Botucatu-SP, Brazil. Interpretation of data, technical procedures.

${ }^{\text {III } P h D, ~ A s s i s t a n t ~ P r o f e s s o r, ~ D e p a r t a m e n t ~ o f ~ S u r g e r y ~ \& ~ O r t h o p e d i c s, ~ F a c u l t y ~ o f ~ M e d i c i n e, ~ U N E S P, ~ B o t u c a t u-S P, ~ B r a z i l . ~ A c q u i s i t i o n ~ o f ~ d a t a, ~ m a n u s c r i p t ~ w r i t i n g . ~}$

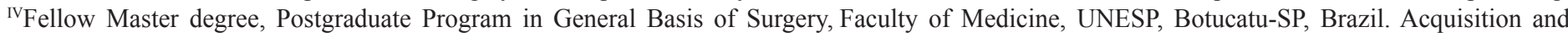
interpretation of data.

Text

\section{ABSTRACT}

PURPOSE: To evaluate morphological changes of the gastric stump and not resected stomach mucosa after the completion of truncal vagotomy.

METHODS: Eighty male Wistar rats were divided into four groups: CT, TV, RY and RYTV. In CT group, abdominal viscera were manipulated and the abdominal cavity was closed, in TV vagal trunks were isolated and sectioned, in RY a partial Roux-en-Y gastrectomy was performed and in RYTV the vagal trunks were sectioned and a partial Roux-en-Y gastrectomy was performed. At the 54th week after surgery, the rats were euthanized. The findings were submitted to histological analyses.

RESULTS: None macroscopic or histological alterations in groups TV and CT was observed. Specimens from RY and RYTV groups did not show alterations in the gastric stump mucosa. At the jejunal side of the gastroenterostomy we found shallow ulcerative lesions always single, well-defined and with variable diameter 3 to $6 \mathrm{~mm}$, six times in the RY group and none in the RYTV group (RY>RYTV, $\mathrm{p}=0.008$ ). Neoplastic or preneoplastic lesions were not diagnosed in all groups.

CONCLUSION: Truncal vagotomy is a safe and non-carcinogenic method in not resected and partially resected stomach.

Key words: Vagotomy, Truncal. Adenocarcinoma. Stomach. Rats. 


\section{Introduction}

Truncal vagotomy is a surgical technique still used nowadays in elective and emergency surgical procedures ${ }^{1-3}$. For patients presenting with complicated peptic ulcer disease such as perforation and /or hemorrhage not controlled by endoscopic and clinical treatment, truncal vagotomy associated with a drainage procedure is a fast and effective treatment. This technique can also be used in elective situations as a complement to reducing acid in distal gastrectomies or when the surgeon chooses the reconstruction of alimentary tract through a Roux-en-Y partial gastrectomy. In Roux-en-Y procedure there is a risk of postoperative development of anastomotic ulcers at jejunal side of gastroenterostomy, caused by diversion of biliopancreatic secretions from the gastroenterostomy and therefore damaging the buffering of the chlorydropeptic secretion produced by the stomach remnant. Moreover, truncal vagotomy has been identified as a risk factor for the development of gastric cancer in epidemiological and experimental studies ${ }^{4-10}$. The mucosa denervation would lead to a decrease in the gastric mucosa cell function, cytoprotection, and an increase in regeneration of immature cells ${ }^{8}$.

\section{Methods}

The study was developed after approval of the Ethics Committee of Experimental Research of UNESP (Protocol 612) and follows the Council for International Organization of Medical Sciences (CIOMS) ethical code for animal experimentation.

Eighty male Wistar rats weighing approximately 150 grams provided by the Central Animal Facility of the Faculty of Medicine of Botucatu were used. Animals, five in number per unit, were housed in plastic boxes wrapped in room with steam exhaust air, artificial light cycles of 12 hours and room temperature maintained at $24^{\circ} \mathrm{C}$. The animals were offered water ad libitum and standard rat chow. After the end of the adaptation period, they were divided in four experimental groups of 20 rats in each group, as described:

Control (CT): underwent to laparotomy and manipulation of the abdominal viscera.

Truncal vagotomy (TV): underwent to section of the anterior and posterior vagal trunks.

Roux-en-Y (RY): underwent to resection of half of the glandular stomach and Roux-en-Y gastroenterostomy.

Roux-en-Y + Truncal Vagotomy (RYTV): underwent to the same surgical procedure of group RY, adding bilateral truncal vagotomy.
In all surgical procedures, animals received anesthesia by intraperitoneal injection of sodium pentobarbital at a dose of $30 \mathrm{mg} / \mathrm{kg}$ body weight. Access to the peritoneal cavity was obtained by laparotomy with $3 \mathrm{~cm}$ long. In CT group, abdominal viscera were manipulated and the abdominal cavity was closed. In TV group, vagal trunks were dissected, isolated and sectioned. In RY group distal half of the glandular stomach was resected, the duodenal stump was buried. The gastrointestinal transit was reconstituted by a Roux-en-Y gastroenterostomy between the proximal cut edge of afferent loop and the efferent limb $10 \mathrm{~cm}$ below the gastroenterostomy. RYTV group animals were subjected to a combination of the procedures performed in RY and TV groups. All sutures were done with a 6.0 polypropylene (Ethicon). The layout of surgical procedures is illustrated in Figure 1.

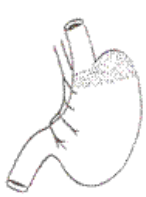

Control

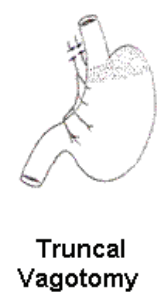

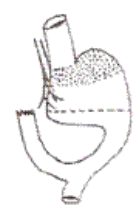

Roux en $Y$

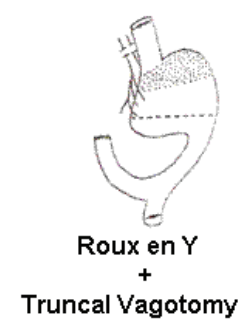

Truncal Vagotomy
FIGURE 1 - Schematic representation of the surgical procedures.

After the surgical procedure, the animals had access to water only on the first $24 \mathrm{~h}$ postoperatively, freeing up access to feed after this period. The control of body weight was done weekly in the morning and deaths recorded. At the 54th week after surgery, the rats were euthanized. They were initially anesthetized with the same methodology used for surgical procedures. Through sternotomy and laparotomy intracavitary organs were checked, the stomach were removed, photographed and fixed in 10\% formalin for $48 \mathrm{~h}$. After fixation, each specimen was sectioned through successive longitudinal sections including the pyloric and oxyntic mucosa and separately squamous epithelium.

At the gastroenterostomy, the sections were perpendicular to it one. We used conventional methods for the preparation of histological slides stained by the hematoxylin/eosin. Histopathological examination was performed using an optical microscope. For the statistical analysis was adjusted a generalized linear model assuming a binomial distribution with LOGIT link. Differences were considered significant when the $\mathrm{p}<0.05$. 


\section{Results}

The effective number of animals in each group was $\mathrm{CT}=$ $20, \mathrm{TV}=20, \mathrm{RY}=13$ and $\mathrm{RYTV}=12$. Postoperative complications, malnutrition and intra-abdominal abscesses were the causes of 15 deaths recorded during the experiment. The examination of the abdominal cavity revealed no significant changes.

The examination of the surgical specimens of CT and TV groups showed no significant gross lesions in both glandular and squamous portion of stomach.

The macroscopic appearance of specimens of the RY and RYTV groups did not show alterations in the squamous portion and in the gastric stump mucosa. The general appearance of specims from RYTV is illustrated in Figure 2A. At the jejunal side of the gastroenterostomy we found shallow ulcerative lesions always single, well-defined, variable diameter 3 to $6 \mathrm{~mm}$, six times in the RY group and none in the RYTV group (RY $>$ RYTV, p = 0.008), (Figure 2B).
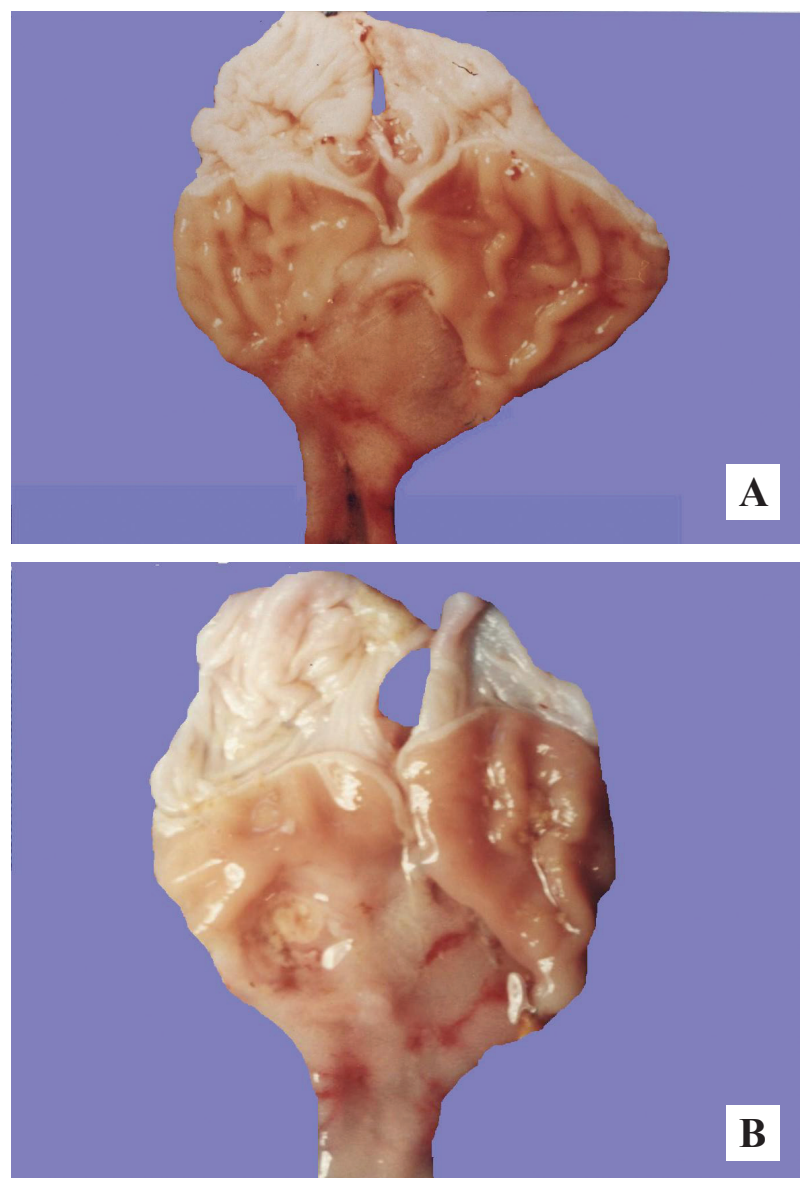

FIGURE 2 - Macroscopic aspect of the specims from RYTV at the $54^{\text {th }}$ week (A). Ulcerated lesion located in the jejunal side of gastroenterostomy (black arrows) in specimen from RY at the $54^{\text {th }}$ week (B). Sq $=$ Squamous portion; $\mathrm{Gl}=$ Glandular portion; $\mathrm{J}=$ Jejunum.
Histological analyses of ulcerated lesions showed that the bottom of the lesions consisted of fibrin crust, variable inflammatory infiltrate and proliferation of collagen. There was no cellular atypia to suggest malignancy (Figure 3). None other significant histological alteration was observed in all groups.

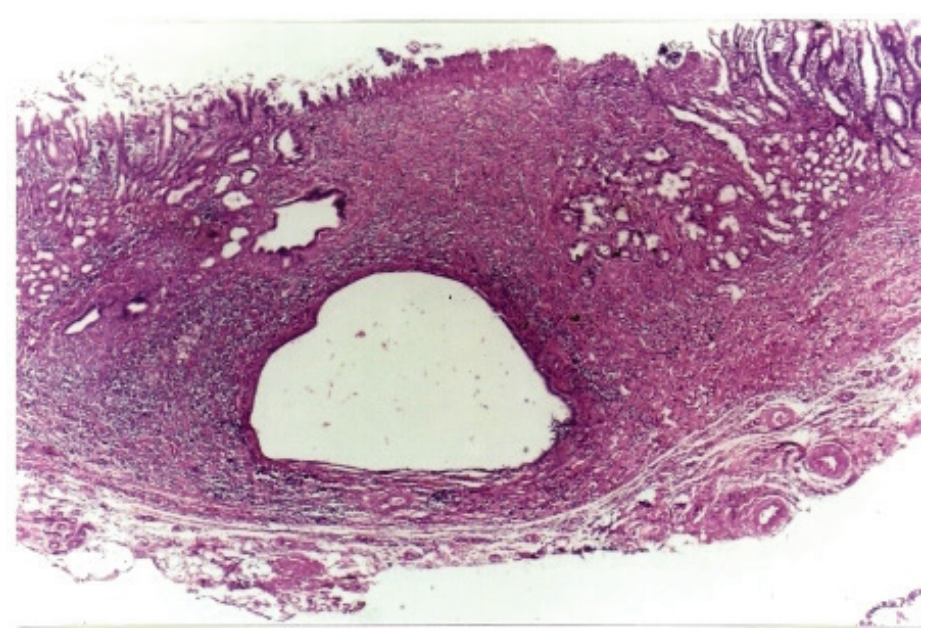

FIGURE 3 - Ulcerated lesion in the jejunal side of gastroenterostomy (black arrows) showing proliferation of collagen and mild inflammatory process, (H\&E x40).

\section{Discussion}

The only significative difference observed among the groups was the occurrence of ulcerative lesions in the jejunal side of the gastroenterostomy, observed in six specimens of the RY group. These lesions may have been caused by the contact between chlorydropeptic secretions, produced in the gastric stump, with the jejunal mucosa. Roux-en-Y procedure causes a decrease in the release of hormones such as secretin and cholecystokinin that, in addition to stimulating the release of pancreatic secretion and gallbladder contraction, results in inhibition of gastric secretion ${ }^{11,12}$. Furthermore, in this type of surgery, the chlorydropeptic secretion produced in the gastric stump is not buffered by the neutralizing effect of biliopancreatic secretions, which transits the gastroenterostomy, as occurs in the Billroth II gastrectomies. The truncal vagotomy was proved effective in preventing the development of gastroenterostomy ulcers in animals that underwent Roux-en-Y partial gastrectomy. Previous studies also showed the same results ${ }^{13,14}$. A study compared the frequency of anastomotic ulcers in rat's subjected to partial Roux-en-Y gastrectomy with or without truncal vagotomy and observed that the occurrence of anastomotic ulcers was significantly higher in the group without vagotomy $(92.8 \%$ versus $14.3 \%)$ after a ten months follow up ${ }^{13}$. Another 
study in pigs submitted to a Roux-en-Y gastrectomy, with or without truncal vagotomy, obtained a $50 \%$ incidence of ulcers in the non-vagotomized and only $20 \%$ in the underwent to a truncal vagotomy ${ }^{14}$. In the present study, it was not observed the development of benign or malignant proliferative lesions in intact stomach or gastric stump mucosa. However, we cannot fail to mention that truncal vagotomy has the potential to facilitate the development of such lesions. Truncal vagotomy causes several changes in gastric physiology since it decreases the ability of the stomach to secrete hydrochloric acid causing an increase in intragastric $\mathrm{pH}$ and consequently the formation of carcinogenic $\mathrm{N}$-nitroso compounds ${ }^{15}$. TV may also change the permeability of the mucosa of the stomach due to decreased in secretion of neutral mucins and lose of regulation of submucosal blood flow, facilitating the contact of the N-nitroso compounds with the proliferative compartment of gastric epithelium ${ }^{16}$.

Some epidemiological studies suggest that vagotomy can increase the risk of gastric cancer development. In one of them, the authors found an increase risk of gastric cancer development 1.6 times higher than expected comparing patients underwent to a vagotomy for treatment of peptic ulcer and controls ${ }^{4,5}$. In another study, which examined cancer incidence in 1,992 patients undergoing to a gastric surgery found that, particularly, the vagotomized had a significantly increased risk of gastric cancer and questions the safety of prolonged acid suppression through use of inhibitors of the proton pump ${ }^{17}$. Some experimental studies also suggest that vagotomy can raise the risk of developing gastric cancer: in one study the author refers to be possible to obtaining gastric cancer in rats subjected to a truncal vagotomy and drainage procedure without using carcinogens ${ }^{6}$; in another study was reported that vagotomy significantly increases the incidence and number of gastric adenocarcinoma obtained from rats treated with $\mathrm{MNNG}^{7}$; and finally one study reports that vagotomy associated with duodenogastric reflux in rats treated with MNNG increases the number of tumors obtained when compared to duodenogastric reflux only, and believe that denervation of the gastric mucosa not only leads to decreased of gastric mucosa cell function and cytoprotection but it also increases the regeneration of immature cells ${ }^{8,18}$.

\section{Conclusion}

Truncal vagotomy is a safe and effective method in not resected and partially resected stomach, since it prevents the development of anastomotic ulcers and does not causes the development of lesions considered preneoplastic or neoplastic.

\section{References}

1. Tsugawa K, Koyanagi N, Hashizume M, Tomikawa M, Akahoshi K, Ayuama K, Wada H, Tandue K, Sugimachi K. The therapeutic strategies in performing emergency surgery for gastroduodenal ulcer perforation in 130 patients over 70 year of age. Hepatogastroenterology. 2001;48:156-62.

2. Jani K, Saxena A, Kothari A. Laparoscopic truncal vagotomy with gastrojejunostomy. J Indian Med Assoc. 2010;108(10):648-51.

3. Wang YR, Richter JE, Dempsey DT. Trends and outcomes of hospitalizations for peptic ulcer disease in the United States, 1993 to 2006. Ann Surg. 2010;251(1):51-8

4. Caygill CP, Hill MJ, Kirkham JS, Northfield TC. Mortality from gastric cancer following gastric surgery for peptic ulcer. Lancet. 1986;1(8487):929-31.

5. Caygill CP, Knowles RL, Hall R. Increased risk of cancer mortality after vagotomy for peptic ulcer: a preliminary analysis. Eur J Cancer Prev. 1991;(1):35-7.

6. Stegemann B. Carcinogenesis following vagotomy. Experimental long-term animal studies. Fortschr Med. 1982;100(13):586-91.

7. Tatsuta M, Yamamura H, Iishi H, Ichii M, Noguchi S, Baba M, Taniguchi $\mathrm{H}$. Promotion by vagotomy of gastric carcinogenesis induced by N-methyl-N'-nitro-N-nitrosoguanidine in Wistar rats. Cancer Res. 1985;45(1):194-7.

8. Kaminishi M, Oohara T, Chiu ML, Aoki F, Yamaguchi H, Shimoyama S. Severe gastric mucosal changes following vagotomy with duodenogastric reflux. J Clin Gastroenterol. 1992;14(Suppl. 1):515-24.

9. Bahmanyar S, Ye W, Dickman PW, Nyrén O. Long-Term Risk of Gastric Cancer by Subsite in Operated and Unoperated Patients Hospitalized for Peptic Ulcer. Am J Gastroenterol. 2007;102:1185-91.

10. Rodrigues PA, Kobayasi S, Rodrigues MAM. Does troncular vagotomy modify the proliferative gastric lesions induced in rats by duodenogastric reflux? Acta Cir Bras. 2007;22(3):210-4.

11. Dragstedt LR, Woodward ER, Seito T, Isaza J, Rodriguez JR, Samilan R. The question of bile regurgitation as cause of gastric cancer. Ann Surg .1971;174:548-59.

12. Hoya Y, Mitsumori N, Yanaga K. The Advantages and disadvantages of a Roux-en-Y reconstruction after a distal gastrectomy for gastric cancer. Surg Today. 2009;39:647-51.

13. Arlt G, Schumpelick V, Riechert B, Klopel G. The ulcer risk in reflux preventive gastric operations. An experimental study. Scand J Gastroenterol. 1984;19:125-8.

14. Walgenbach S, Junginger TH, Hage C, Wolfinger S, Schollmeyer J. Tiexperimentelle unter suchungen zur genese von anstomosenulzena nach reflux belasteter und reflux verhutender magenressection. Langebachs Arch Chir. 1991;376:69-76.

15. Correa P, Haenzel W, Cuello C, Tannenbaum S, Archer MA. Model for gastric cancer epidemiology. Lancet. 1975;11:58-60.

16. Kaminishi M, Shimizu N, Shiomayama S, Yamaguchi H, Ogawa T, Sakai S, Kuramoto S, Oohara T. Etiology of gastric remnant cancer with special reference to the effects of denervation of the gastric mucosa. Cancer Supplement. 1995;75:1490-96.

17. Jenkins JT, Duncan JR, Hole D, O'dwyer PJ, McGregor JR. Malignant disease in peptic ulcer surgery patients after long term follow-up: A cohort study of 1992 patients. Eur J Surg Oncol. 2007;33:766-12.

18. Landa GJL, Carabias HA, Rodriguez DS, Alcalde EJ, Ortega ML, Balibrea CJL. Protective effect of vagotomy on the gastric mucosa in a stress model in rats. Rev Esp Enferm Dig. 2002;94(12):737-44. 


\section{Acknowledgement}

To Professor César Tadeu Spadella by a critical review of the study, formatting and proofreading the manuscript in English.

\section{Correspondence:}

Paulo Antônio Rodrigues

Alameda das Grevílias, 300

18607-360 Botucatu - SP Brasil

Tel.:(5514)3880-1435

paulor@fmb.unesp.br

Received: Oct 24, 2013

Review: Dec 20, 2013

Accepted: Jan 21, 2014

Conflict of interest: none

Financial source: none

${ }^{1}$ Research performed at Laboratory of Surgical Technique and Experimental Surgery, Faculty of Medicine, Sao Paulo State University (UNESP), Botucatu-SP, Brazil 